\title{
An alternative approach to decompose the potential gains from mergers
}

\author{
Liang Liang ${ }^{\mathrm{a}}$, Feng $\mathrm{Li}^{\mathrm{a}}$, Yongjun $\mathrm{Li}^{\mathrm{a}, *}$, Ali Emrouznejad ${ }^{\mathrm{b}}$ \\ ${ }^{a}$ School of Management, University of Science and Technology of China, Hefei, Anhui 230026, P.R. China \\ ${ }^{b}$ Aston Business School, Aston School, Birmingham, UK
}

\begin{abstract}
Bogetoft and Wang (2005) proposed admirable production economic models to estimate and decompose the potential gains from mergers. They provided a good platform to quantify the merger efficiency and related it to relevant organizational changes ex-ante. In this paper, we develop an alternative approach to decompose the potential overall gains from mergers into to technical effect, size effect and harmony effect. The proposed approach uses strongly efficient projections, and consistently calculates radial input based measures of these three effects based on the pre-merger aggregated inputs. In addition, the proposed approach is of vital significance in two special cases where the aggregated projected inputs are not proportional to the pre-merger aggregated inputs and where the production sizes are very different for the original decision making units (DMUs). Finally, an application to the City Commercial Banks (CCBs) in China is provided to illustrate the usefulness and efficacy of the proposed approach. The application shows that there exist significant merger efficiency gains for these top $20 \mathrm{CCBs}$. Further, both the technical effect and harmony effect favor mergers, whereas the size effect would work against most mergers. Thus, in most cases the full-size merger with "organizational sense" is not proper.
\end{abstract}

Keywords: Data Envelopment Analysis (DEA); Merger efficiency; Decomposition

\footnotetext{
* Corresponding author. Tel: +86 55163606822 .

E-mail addresses: 1liang@ustc.edu.cn (L. Liang), lfeng90@mail.ustc.edu.cn (F. Li), lionli@ustc.edu.cn (Y.Li), a.emrouznejad@aston.ac.uk (A. Emrouznejad).
} 


\section{Introduction}

Mergers, which play an important role in today's economy life, has proven to be one of the most successful and simplest ways to strengthen firms' advantages, improve their performance, and ensure their continued survival. Extensive literature has been published on mergers, some of which tries to quantify the potential gains of mergers. Prime contributions were made by Bogetoft et al. (2003) and Bogetoft and Wang (2005) (hereafter BW), where they focused on the potential production economic effects and proposed admirable production economic models to estimate and decompose the potential gains from mergers. Based on their rationale, Ray (2004), Bagdadioglu et al. (2007), Kristensen et al. (2010), Blancard et al. (2011, 2016), Wu and Birge (2012), Peyrache (2013), Wu et al. (2011, 2014), and Shi et al. (2017) worked deeper on this issue in many different contexts. So far, the rationale proposed by $\mathrm{BW}$ has been regarded as the basis of estimation and decomposition of the potential gains ex-ante.

By identifying means to improve performance and estimate particular gains, BW provided a good platform to decompose the potential overall gains into technical effect, harmony effect and size effect. In this paper, we develop an alternative approach to decompose the potential gains from mergers. The proposed approach uses strongly efficient projections instead of weakly efficient projections, and consistently calculates radial measures based on the pre-merger aggregated inputs. Besides, the proposed approach is supposed to be of vital significance in two special cases, where the aggregated projected inputs are not proportional to the pre-merger aggregated inputs and where the output production sizes are very different for the original decision making units (DMUs). From this perspective, we extend the decomposition framework proposed by BW to wider applications.

The remainder of this paper is organized as follows: In Section 2, we briefly introduce some preliminaries and suggest the usage of strongly efficient projections. Section 3 proposes an alternative decomposition approach and compares it with the existing approach. An application to the City Commercial Banks (CCBs) in China is 
provided in Section 4 to demonstrate the usefulness of the proposed approach. Finally, Section 5 concludes this paper and provides directions for future research.

\section{Preliminaries}

Following a common framework in data envelopment analysis (DEA) literature, we assume that there are $n$ peer DMUs that consume $m$ inputs $X_{j}=\left(x_{1 j}, \ldots, x_{m j}\right)^{T}$ to produce $s$ outputs $Y_{j}=\left(y_{1 j}, \ldots, y_{s j}\right)^{T}$. To calculate the technical efficiency score of $D M U_{d}(d=1, \ldots, n)$, we can calculate the following model (1):

$$
\begin{aligned}
\theta_{d}^{*}= & \operatorname{Min} \theta_{d} \\
\text { s.t. } & \sum_{j=1}^{n} \lambda_{j} X_{j} \leq \theta_{d} X_{d} \\
& \sum_{j=1}^{n} \lambda_{j} Y_{j} \geq Y_{d} \\
& \lambda_{j} \geq 0, \lambda_{j} \in \Omega, j=1, \ldots, n .
\end{aligned}
$$

Here $\lambda_{j} \in \Omega$ represents the constraint imposed on the intensity variables, which are used to construct the production frontier. For example, $\lambda_{j} \in \Omega$ can be formulated as $\quad \sum_{j=1}^{n} \lambda_{j}>1, \quad \sum_{j=1}^{n} \lambda_{j}=1$ and $\sum_{j=1}^{n} \lambda_{j}<1$ for decreasing, variable, and increasing returns to scale properties, respectively. If we only concern the non-negative requirement $\lambda_{j} \geq 0$, then model (1) is changed into the original CCR-DEA model (Charnes et al., 1978). The optimal solution of $\theta_{d}$, namely, $\theta_{d}^{*}$, implies the maximal proportional contraction in inputs while keeping outputs unchanged. The value of $\theta_{d}^{*}$ is taken as the efficiency score of $D M U_{d}(d=1, \ldots, n)$. $D M U_{d}(d=1, \ldots, n)$ is identified as DEA efficient if $\theta_{d}^{*}$ reaches the maximum of one, otherwise $D M U_{d}(d=1, \ldots, n)$ is inefficient if $\theta_{d}^{*}<1$.

Suppose DMUs with subscripts $j \in J \subseteq\{1, \ldots, n\}$ are merged to be a new unit, then the aggregated inputs and outputs are $\sum_{j \in J} X_{j}$ and $\sum_{j \in J} Y_{j}$, respectively. To estimate the potential overall gains from mergers, Bogetoft and Wang (2005) suggested the following model (2): 


$$
\begin{aligned}
E^{J}= & \operatorname{Min} E \\
\text { s.t. } & \sum_{j=1}^{n} \lambda_{j} X_{j} \leq E \cdot \sum_{j \in J} X_{j} \\
& \sum_{j=1}^{n} \lambda_{j} Y_{j} \geq \sum_{j \in J} Y_{j} \\
& \lambda_{j} \geq 0, \lambda_{j} \in \Omega, j=1, \ldots, n .
\end{aligned}
$$

Here the efficient frontier is assumed to be constructed only by the original DMUs. The radial input based measure $E^{J}$ indicates the maximal reduction on the aggregated inputs $\sum_{j \in J} X_{j}$ when producing the aggregated outputs $\sum_{j \in J} Y_{j}$. If $E^{J}<1$, the merger is advantageous, whereas if $E^{J}>1$ the merger is costly. More specially, this program is similar with the super-efficiency concept (Andersen and Petersen, 1993), which excludes the evaluated DMU J from the reference set, hence it may be infeasible. By definition, it holds $E^{J}=+\infty$ if this program is infeasible. Model (2) is input-oriented. The output-oriented potential overall gains calculations and decompositions are similar to the input oriented, therefore, we focus only on the input oriented case throughout this paper.

Aiming to investigating the production effects from mergers, Bogetoft and Wang (2005) introduced admirable production economic models. For the convenience of reading, we briefly summarize the BW decomposition in Appendix A. Here in this paper, given the potential overall gains defined in model (2), we will propose an alternative approach to decompose the potential gains from mergers. Before doing so, we suggest the usage of strongly efficient projections. If strongly efficiency projections are used to measure the efficiency gain by individual units as they move to the merger frontier, then a different figure for the technical efficiency component and pure potential gains results, and consequently, given that the decomposition is an identity, the remaining harmony and size measures must change.

Here we take the BW decomposition for example to highlight the difference derived from strongly efficient projections and weakly efficient projections. There are five DMUs (i.e., A, C, E, F and B), with each using two inputs $x_{1}$ and $x_{2}$ to generate the same output $y$. They construct the efficient frontier $L(y)=A^{\prime} C E F B^{\prime}$, where $\mathrm{A}^{\prime}$ is a point that line $\mathrm{OA}$ crosses $\mathrm{CA}^{\prime}$ (a parallel of axis $x_{2}$ ), and $\mathrm{B}^{\prime}$ is a point between $\mathrm{OB}$ and FB' (a parallel of axis $x_{1}$ ). 


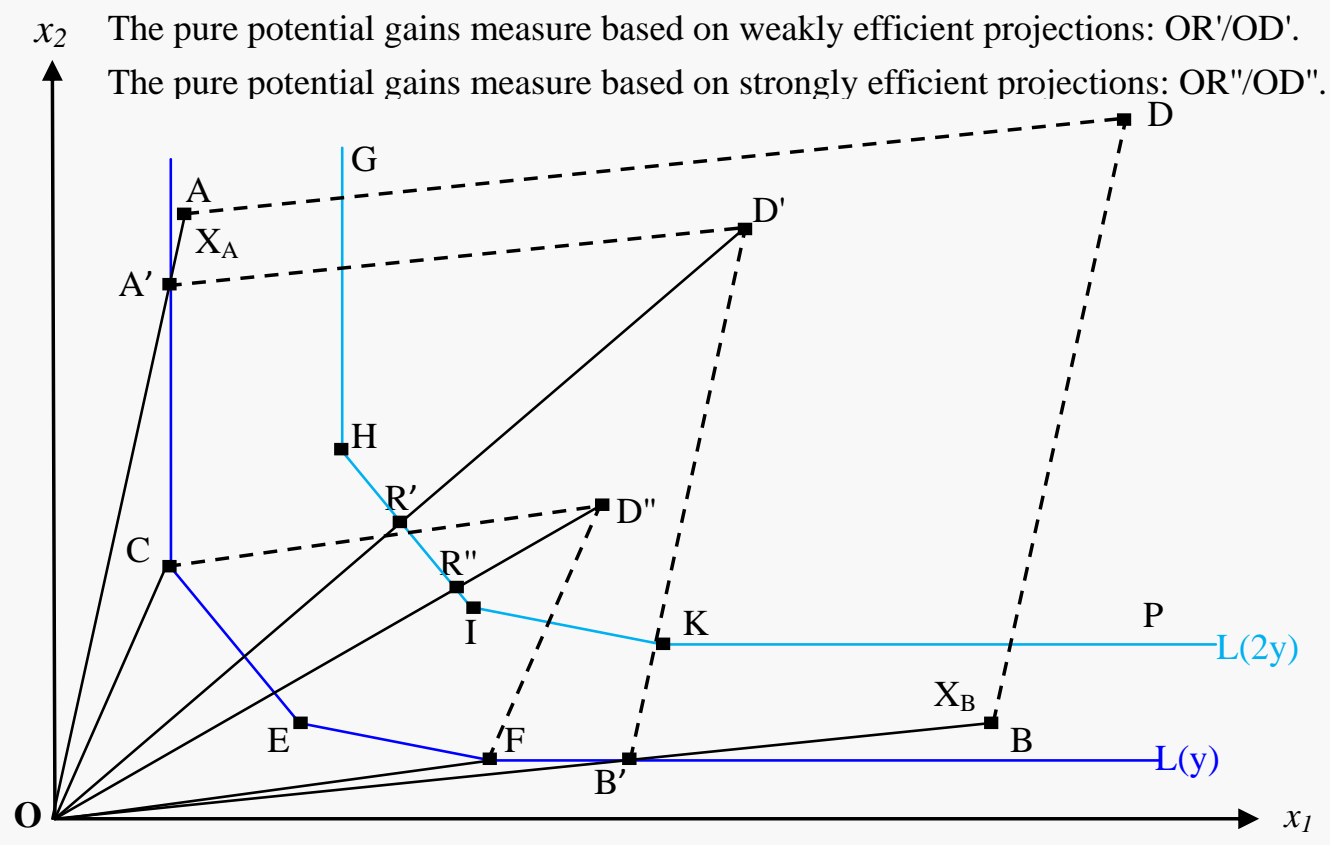

Fig. 1 The pure potential gains based on different projections

According to model (1), DMU A and B are inefficient, and C, E and F are efficient. Suppose $\mathrm{DMU}_{\mathrm{A}}$ and $\mathrm{DMU}_{\mathrm{B}}$ 's efficiencies are $\theta_{A}^{*}$ and $\theta_{B}^{*}$, respectively, therefore, $A^{\prime}=\left(\theta_{A}^{*} X_{A}, y\right)$ and $B^{\prime}=\left(\theta_{B}^{*} X_{B}, y\right)$ are weakly efficient projections of $\mathrm{DMU}_{\mathrm{A}}$ and $\mathrm{DMU}_{\mathrm{B}}$, respectively. On the contrary, according to Cooper et al. (2011, p45), their strongly efficient ones should be $\mathrm{DMU}_{\mathrm{C}}$ and $\mathrm{DMU}_{\mathrm{F}}$ such that $C=\left(\theta_{A}^{*} X_{A}-\left(\begin{array}{c}0 \\ x_{2 A^{\prime}}-x_{2 C}\end{array}\right), y\right)$ and $F=\left(\theta_{B}^{*} X_{B}-\left(\begin{array}{c}x_{1 B^{\prime}}-x_{1 F} \\ 0\end{array}\right), y\right)$, respectively.

Different projections used in Fig. 1 lead to different pure potential gains. For convenience of understanding this point, we introduce the efficient frontier $L(2 y)=G H I K P$ for the output level $2 y$, where D' is the sum of weakly efficient projections $\mathrm{A}^{\prime}$ and $\mathrm{B}^{\prime}, \mathrm{D}$ " is the sum of strongly efficient projections $\mathrm{C}$ and F. In Fig. 1, line $\mathrm{OD}^{\prime}$ and $\mathrm{OD}^{\prime \prime}$ cross $L(2 y)$ at point $\mathrm{R}^{\prime}$ and $\mathrm{R}^{\prime \prime}$, respectively. According to the $\mathrm{BW}$ calculation, the pure potential gains can be calculated as a ratio $E^{* J}=O R^{\prime} / O D^{\prime}$. However, if strongly efficient projections are used, it would be $\hat{E}^{* J}=O R " / O D "$. The two pure potential gains measures have a relation via a following theorem:

Theorem 1. The pure potential gains measure based on weakly efficient projections is 
no more than that based on strongly efficient projections, that is, $E^{* J} \leq \hat{E}^{* J}$.

Proof: See Appendix B.

As a higher measure implies a smaller gain from mergers, Theorem 1 shows that the usage of strongly efficient projections would owe less to the pure potential gains and more to the technical effect component, as compared to weakly efficient projections.

\section{New models}

For the sake of using strongly efficient projections, here we first introduce model (3).

$\operatorname{Max} 1 \delta_{d}$

$$
\begin{array}{ll}
\text { s.t. } & \sum_{j=1}^{n} \lambda_{j} X_{j}=\theta_{d}^{*} X_{d}-\delta_{d} \\
& \sum_{j=1}^{n} \lambda_{j} Y_{j} \geq Y_{d} \\
& \delta_{d} \geq \mathbf{0} \\
& \lambda_{j} \geq 0, \lambda_{j} \in \Omega, j=1, \ldots, n .
\end{array}
$$

where $\theta_{d}^{*}$ is the efficiency score calculated by model (1) and $\delta_{d}=\left(\delta_{1 d}, \ldots, \delta_{m d}\right)^{T}$ is the non-negative vector of input slacks for $\operatorname{DMU}_{d}(d=1, \ldots, n)$. Suppose the optimal solution gets $\delta_{d}^{*}=\left(\delta_{1 d}^{*}, \ldots, \delta_{m d}^{*}\right)^{T}$, then we obtain the strongly efficient projection (Cooper at al., 2011, p45) for each $D M U_{j}(j=1, \ldots, n)$ as given by Formula (4).

$$
\left(\theta_{j}^{*} X_{j}-\delta_{j}^{*}, Y_{j}\right)
$$

Then, for a given merged DMU J, the sum of its members' strongly efficient projections can be expressed as

$$
\left(\sum_{j \in J}\left(\theta_{j}^{*} X_{j}-\delta_{j}^{*}\right), \sum_{j \in J} Y_{j}\right)
$$

To calculate the radial input based measure of technical effect, we propose the following model (6).

$$
T^{J}=\operatorname{Min}\left\{T \in R^{+} \mid \sum_{j \in J}\left(\theta_{j}^{*} X_{j}-\delta_{j}^{*}\right) \leq T \cdot \sum_{j \in J} X_{j}\right\}
$$

Model (6) calculates an optimal proportional reduction based on the pre-merger aggregated inputs $\sum_{j \in J} X_{j}$ (the reason can be found in the second part "consistent radial measures" in Section 3.1). In the one-dimension case, both $\sum_{j \in J}\left(\theta_{j}^{*} X_{j}-\delta_{j}^{*}\right)$ 
and $\quad \sum_{j \in J} X_{j}$ are scalars and the $\mathrm{T}^{\mathrm{J}}$ can be calculated as $T^{J}=\sum_{j \in J}\left(\theta_{j}^{*} X_{j}-\delta_{j}^{*}\right) / \sum_{j \in J} X_{j}$. In the multi-dimension case, however, ratios of $\sum_{j \in J}\left(\theta_{j}^{*} x_{i j}-\delta_{i j}^{*}\right) / \sum_{j \in J} x_{i j}, i=\{1, \ldots, m\}$ may vary heavily. Model (6) selects the maximum of all $\sum_{j \in J}\left(\theta_{j}^{*} x_{i j}-\delta_{i j}^{*}\right) / \sum_{j \in J} x_{i j}, i=\{1, \ldots, m\}$ as the technical effect measure. And then $\sum_{j \in J}\left(\theta_{j}^{*} X_{j}-\delta_{j}^{*}\right) \leq T^{J} \cdot \sum_{j \in J} X_{j}$ would hold equality for some dimensions and inequality for the others, which implies that the inequality-held inputs can be further reduced, but an additional proportional reduction on $\sum_{j \in J} X_{j}$ is infeasible as equality-held inputs cannot be reduced anymore.

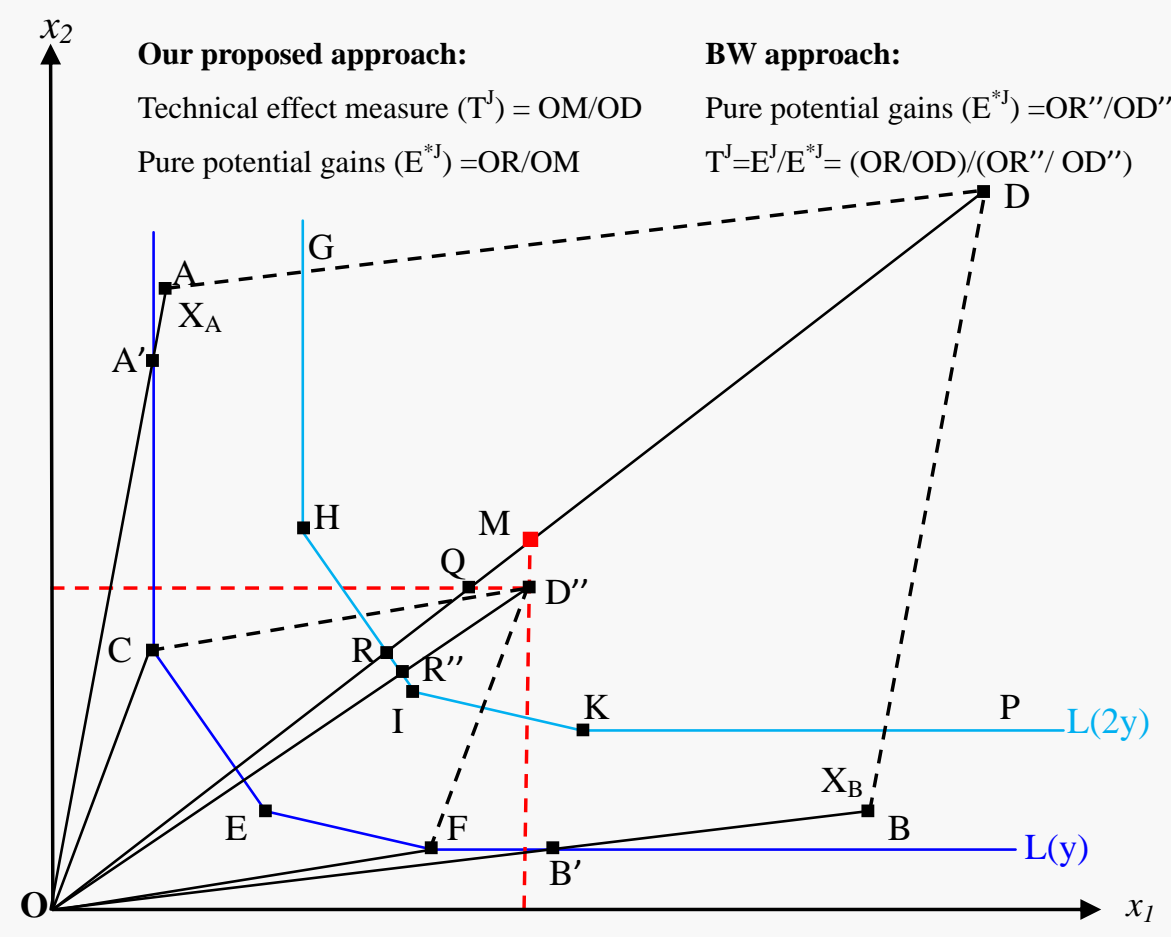

Fig. 2 The decomposition of technical effect and pure potential gains

Here we illustrate model (6) in Fig. 2 where two inefficient DMUs (i.e., $\mathrm{DMU}_{\mathrm{A}}$ and $\left.\mathrm{DMU}_{\mathrm{B}}\right)$ are merged to be a new unit. We use efficiency scores obtained by model (1) to project the original $\mathrm{A}$ and $\mathrm{B}$ to $\mathrm{A}^{\prime}$ and $\mathrm{B}^{\prime}$, respectively. Further, we consider using the optimal input slacks obtained by model (3) to $\mathrm{C}$ and F, respectively, to get the aggregated projected inputs as in point $\mathrm{D}^{\prime \prime}$. Herein line OD" represents the aggregated projected inputs and line OD represents the pre-merger aggregated inputs. To obtain a 
radial technical effect measure based on $\left(X_{A}+X_{B}\right)$, model (6) has been used to project $\mathrm{OD}^{\prime \prime}$ onto OD, which is realized by the two red dotted lines for two inputs in Fig. 2. Finally, $\mathrm{D}^{\prime \prime}$ is projected vertically to $\mathrm{M}$ and horizontally to $\mathrm{Q}$. For input $\mathrm{x}_{1}$ and $\mathrm{x}_{2}$, we get $\mathrm{OM} / \mathrm{OD}$ and $O Q / O D$, respectively, then the maximum $\mathrm{OM} / \mathrm{OD}$ is taken as the radial input based measure of technical effect.

Based on Formula (A1) and (A2), the pure potential gains $\left(E^{* J}\right)$ is calculated by

$$
E^{* J}=E^{J} / T^{J}
$$

In Fig. 2, we calculate the potential overall gains by OR/OD, and the technical effect measure by OM/OD, thus the pure potential gains can be measured by OR/OM.

Further, we address the decomposition of size effect and harmony effect. We first obtain the strongly efficient projection for each $D M U_{j}(j \in J)$ that intends to produce the pre-merger aggregated outputs $\sum_{j \in J} Y_{j}$. Model (8) is formulated to minimize the proportional expansion on $\left(\theta_{j}^{*} X_{j}-\delta_{j}^{*}\right)$,

$$
\begin{aligned}
S_{j}^{*}= & \operatorname{Min} S_{j} \\
\text { s.t. } & \sum_{j=1}^{n} \lambda_{j} X_{j} \leq S_{j} \cdot\left(\theta_{j}^{*} X_{j}-\delta_{j}^{*}\right) \\
& \sum_{j=1}^{n} \lambda_{j} Y_{j} \geq \sum_{j \in J} Y_{j} \\
& \lambda_{j} \geq 0, \lambda_{j} \in \Omega, j=1, \ldots, n .
\end{aligned}
$$

Based on the optimal solution $S_{j}^{*}$ in model (8), we use model (9) to obtain the optimal input slack vector $\beta_{j}^{*}=\left(\beta_{1 j}^{*}, \ldots, \beta_{m j}^{*}\right)^{T}$.

$$
\begin{array}{ll}
\operatorname{Max} \mathbf{1} \beta_{j} \\
\text { s.t. } & \sum_{j=1}^{n} \lambda_{j} X_{j}=S_{j}^{*} \cdot\left(\theta_{j}^{*} X_{j}-\delta_{j}^{*}\right)-\beta_{j} \\
& \sum_{j=1}^{n} \lambda_{j} Y_{j} \geq \sum_{j \in J} Y_{j} \\
& \beta_{j} \geq \mathbf{0}, j=1, \ldots, n \\
& \lambda_{j} \geq 0, \lambda_{j} \in \Omega, j=1, \ldots, n .
\end{array}
$$

In this way, the strongly efficient projection for each $D M U_{j}(j \in J)$ that produces the pre-merger aggregated outputs $\sum_{j \in J} Y_{j}$ can be expressed by Formula (10). 


$$
\left(S_{j}^{*} \cdot\left(\theta_{j}^{*} X_{j}-\delta_{j}^{*}\right)-\beta_{j}^{*}, \sum_{j \in J} Y_{j}\right)
$$

It means that $D M U_{j}(j \in J)$ can alone use total inputs of $S_{j}^{*} \cdot\left(\theta_{j}^{*} X_{j}-\delta_{j}^{*}\right)-\beta_{j}^{*}$ to produce $\sum_{j \in J} Y_{j}$. Therefore, the average production unit $\left(|J|^{-1} \sum_{j \in J}\left(S_{j}^{*} \cdot\left(\theta_{j}^{*} X_{j}-\delta_{j}^{*}\right)-\beta_{j}^{*}\right), \sum_{j \in J} Y_{j}\right)$ can be used to calculate the harmony effect measure, as suggested by Bogetoft and Wang (2005). However, this practice will cause an inconsistent problem where the harmony effect measure is not calculated based on the pre-merger aggregated inputs (the detailed explanation of consistent measures can be found in the second part of Section 3.1). Therefore, to obtain a consistent radial input based measure all the time, we suggest to firstly use model (11) to calculate the size effect measure directly, and all the remaining is attributed to the harmony effect measure.

$$
S^{J}=\operatorname{Min}\left\{\left.S \in R^{+}|| J\right|^{-1} \sum_{j \in J}\left[S_{j}^{*} \cdot\left(\theta_{j}^{*} X_{j}-\delta_{j}^{*}\right)-\beta_{j}^{*}\right] \leq S \cdot T^{J} \sum_{j \in J} X_{j}\right\}
$$

Lastly, once the two components, technical effect and size effect, are estimated, what remains is the calculation of harmony effect. The harmony effect measure is calculated by model (12):

$$
\begin{aligned}
H^{J}= & \operatorname{Min} H \\
\text { s.t. } & \sum_{j=1}^{n} \lambda_{j} X_{j} \leq H \cdot S^{J} T^{J} \sum_{j \in J} X_{j} \\
& \sum_{j=1}^{n} \lambda_{j} Y_{j} \geq \sum_{j \in J} Y_{j} \\
& \lambda_{j} \geq 0, \lambda_{j} \in \Omega, j=1, \ldots, n .
\end{aligned}
$$

Here we illustrate the calculation of size effect and harmony effect in terms of model (8)-(12) in Fig. 3, where these two mergering units $A=\left(X_{A}, y_{1}\right)$ and $B=\left(X_{B}, y_{2}\right)$ are strongly efficient and possess different production sizes. There are four efficient frontiers associated with different output sizes ${ }^{1}$, namely, $L\left(y_{1}\right)$, $L\left(y_{1} / 2+y_{1} / 2\right), L\left(y_{2}\right)$ and $L\left(y_{1}+y_{2}\right)$. We apply model (8) and (9) to projecting A

\footnotetext{
${ }^{1}$ In Fig. 3, this broken line " $2 \mathrm{~L}\left(\mathrm{y}_{1} / 2+\mathrm{y}_{2} / 2\right)$ " does not represent an efficient frontier, as it is just a double projection of $\mathrm{L}\left(\mathrm{y}_{1} / 2+\mathrm{y}_{2} / 2\right)$. Here we just show some parts for simplification.
} 
and $\mathrm{B}$ to strongly efficient projections, so it suffices to use $\left(S_{A}^{*} X_{A}-\beta_{A}^{*}\right)$ or $\left(S_{B}^{*} X_{B}-\beta_{B}^{*}\right)$ to produce $\left(y_{1}+y_{2}\right)$, as given by formula (10). To running model (11), we apply the two red dotted lines to projecting M onto OD (or the elongated line of OD). $\mathrm{M}$ is projected vertically to $\mathrm{Q}$ and horizontally to $\mathrm{M}^{\prime}$, and for input $x_{1}$ and $x_{2}$, we get $O Q / O D$ and $O M^{\prime} / O D$, respectively. Then model (11) calculates the size effect measure by the larger ratio $O M^{\prime} / O D$. Further, there are still possible improvement from $\mathrm{M}^{\prime}$ to $\mathrm{R}$, thus we apply model (12) to calculating the harmony effect measure by $O R / O M^{\prime}$.

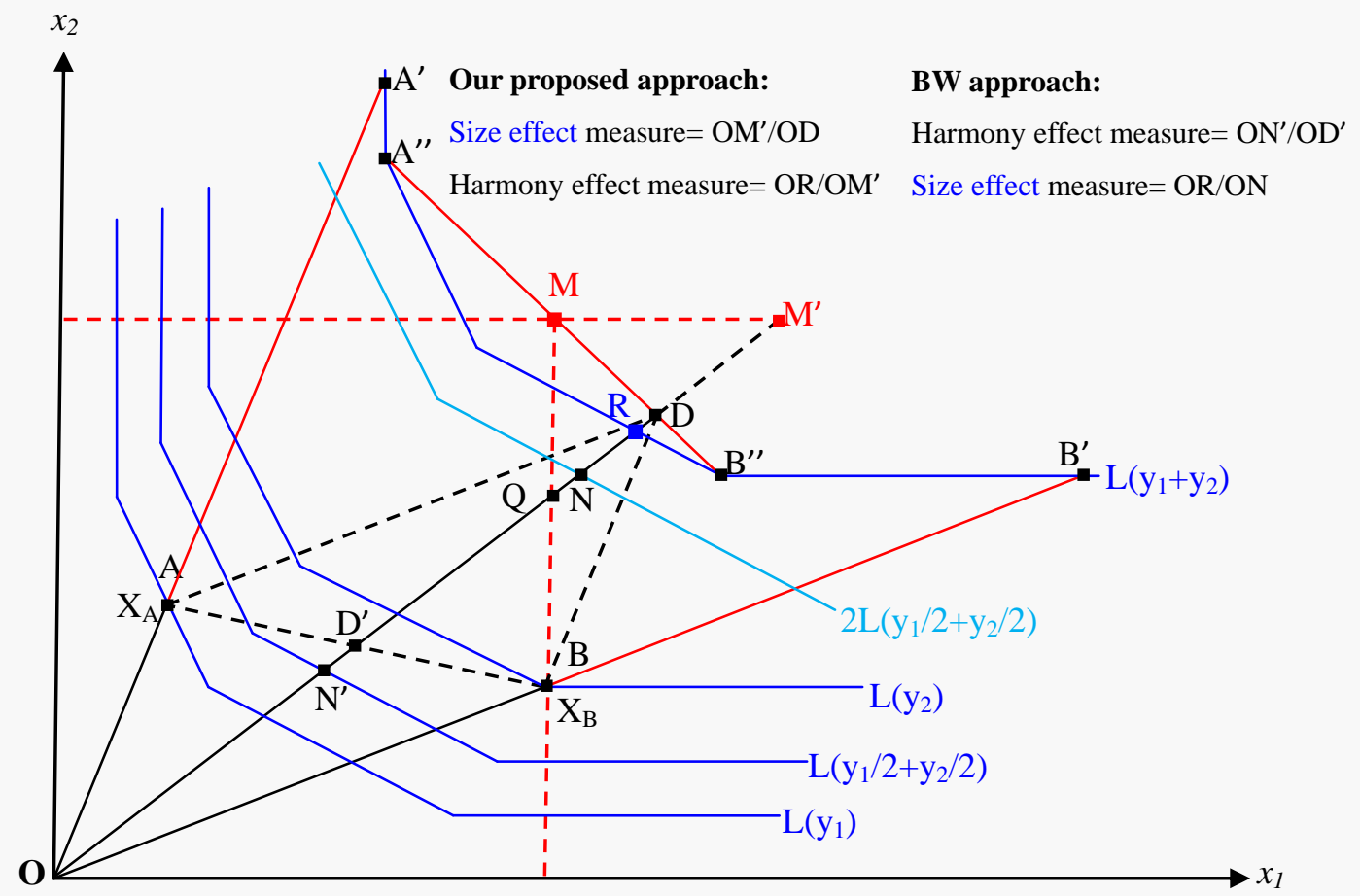

Fig. 3 The decomposition of size effect and harmony effect

\subsection{Comparison between the proposed approach and the BW}

\section{approach}

In this subsection, we compare the proposed approach with the BW approach to highlight some characteristics. Both conceptual and methodological differences of the two approaches have been discussed on: 1) weakly efficient projections vs strongly efficient projections; 2) consistent radial measures; 3) the decomposition of technical effect and pure potential gains, and lastly, 4) the decomposition of size effect and harmony effect. 


\section{1) Weakly efficient projections vs strongly efficient projections}

In this paper we suggest the usage of strongly efficient projections. As discussed previously, if strongly efficiency projections are used to measure the efficiency gains by individual units as they move to the production frontier, then a different figure for the technical efficiency component and pure potential gains results, and consequently, given that the decomposition is an identity, the remaining harmony and size measures must change. Especially, by using strongly efficient projections, many more gains will be attributed to the technical effect and less gains to the pure potential gains from mergers, as compared to weakly efficient projections.

\section{2) Consistent radial measures}

Throughout this paper, our proposed approach gives consistent radial measures. Here the term consistent means that we calculate the measures of these three effects based on the pre-merger aggregated inputs $\sum_{j \in J} X_{j}$, on which the potential overall gains have already been estimated in model (2). This is an important property of our proposed approach. To maximize the radial gains as possible, we adjust merged units to follow the production style of the pre-merger aggregated inputs-outputs, as shown from $D^{\prime \prime}$ to $M$ in Fig. 2 and from $M$ to $M^{\prime}$ in Fig. 3, respectively. As results, it is very easy to relate the proportional input savings to these measures. As given in Table 1, the total input savings is $\left(1-E^{J}\right) \cdot \sum_{j \in J} X_{j}$, with $\left(1-T^{J}\right) \cdot \sum_{j \in J} X_{j}$, $\left(1-S^{J}\right) \cdot T^{J} \sum_{j \in J} X_{j}$ and $\left(1-H^{J}\right) \cdot S^{J} T^{J} \sum_{j \in J} X_{j}$ coming from technical effect, size effect and harmony effect, respectively. However, it is a little difficult for BW to attribute the proportional input savings to relevant effects. On one hand, in the BW approach, the input savings resulting from technical effect is not clear. On the other hand, the BW approach gives inconsistent radial measures. Table 1 shows that the input savings in the last three lines are measured by $\sum_{j \in J} \theta_{j}^{*} X_{j}$ rather than $\sum_{j \in J} X_{j}$, whereas the potential overall gains to be decomposed is proportional to $\sum_{j \in J} X_{j}$ in model (2).

Table 1 Input savings related to individual effects 


\begin{tabular}{lcc}
\hline & The proposed approach & BW approach \\
\hline Potential overall gains & $\left(1-E^{J}\right) \cdot \sum_{j \in J} X_{j}$ & $\left(1-E^{J}\right) \cdot \sum_{j \in J} X_{j}$ \\
Technical effect gains & $\left(1-T^{J}\right) \cdot \sum_{j \in J} X_{j}$ & $?$ \\
Pure potential gains & $\left(1-E^{* J}\right) \cdot T^{J} \sum_{j \in J} X_{j}$ & $\left(1-E^{* J}\right) \cdot \sum_{j \in J} \theta_{j}^{*} X_{j}$ \\
Size effect gains & $\left(1-S^{J}\right) \cdot T^{J} \sum_{j \in J} X_{j}$ & $\left(1-H^{J}\right) \cdot \sum_{j \in J} \theta_{j}^{*} X_{j}$ \\
Harmony effect gains & $\left(1-H^{J}\right) \cdot S^{J} T^{J} \sum_{j \in J} X_{j}$ & $\left(1-S^{J}\right) \cdot H^{J} \sum_{j \in J} \theta_{j}^{*} X_{j}$ \\
\hline
\end{tabular}

\section{3) Decomposition of technical effect and pure potential gains}

Our proposed approach calculates the technical effect measure directly and attributes the residuals to the pure potential gains, and such a practice is still reasonable when the aggregated projected inputs are not proportional to the pre-merger aggregated inputs. We illustrate this special case in Fig. 2, where it holds that $\sum_{j \in J}\left(\theta_{j}^{*} X_{j}-\delta_{j}^{*}\right) \neq k \sum_{j \in J} X_{j}, k \in(0,1]$, that is, three points (i.e., O, D and D") are not collinear. In fact, this is often the case for mergers and acquisitions in real world. As seen in Fig. 2, our proposed approach calculates the technical effect measure by $\mathrm{OM} / \mathrm{OD}$ and it provides a reasonable decomposition. However, in this type of special cases the BW approach may assign a lower value to the technical effect measure as compared to our proposed approach. Note in addition that by eliminating technical inefficiencies, the aggregated input is $\sum_{j \in J}\left(\theta_{j}^{*} X_{j}-\delta_{j}^{*}\right)$. We understand it as the minimal input that can produce the aggregated outputs $\sum_{j \in J} Y_{j}$, and any inputs less than it would be infeasible for technical effect. Here we find that within the special case under consideration, such a lower measure derived from the BW approach represents a proportional input savings that cannot be achieved by eliminating the technical inefficiency. This point can be demonstrated by noting that the relation $\left(E^{J} / E^{* J}\right) \sum_{j \in J} X_{j} \geq \sum_{j \in J}\left(\theta_{j}^{*} X_{j}-\delta_{j}^{*}\right)$ is not held. Readers are referred to Appendix $\mathrm{C}$ for the proof.

In addition, if the aggregated projected inputs are proportional to the pre-merger 
aggregated inputs, our proposed approach obtains the same technical effect measure and pure potential gains as that of BW. We demonstrate it by Theorem 2, where $\mathrm{E}^{* \mathrm{~J}}$ and $\mathrm{T}^{\mathrm{J}}$ are calculated by the $\mathrm{BW}$ approach, $\hat{E}^{* J}$ and $\hat{T}^{J}$ are calculated by our proposed approach.

Theorem 2. If $\sum_{j \in J}\left(\theta_{j}^{*} X_{j}-\delta_{j}^{*}\right)=k \sum_{j \in J} X_{j}, k \in(0,1]$, then $E^{* J}=\hat{E}^{* J}, T^{J}=\hat{T}^{J}$.

Proof: See Appendix D.

\section{4) Decomposition of size effect and harmony effect}

As for the decomposition of size effect and harmony effect, we provide a feasible approach to avoid the mix of these two effects when the output production sizes are very different for the original DMUs. In the BW, it used the average unit to avoid the inclusion of size effect when they estimate the harmony effect gains. However, a possible application condition is that the individual production sizes should not differ a lot, as already suggested by BW themselves. Otherwise, some size effects may be picked up in the harmony effect measure. In real applications, however, almost all firms or entities are different in production sizes. Therefore, the BW approach would indeed present overlaps between harmony effect and size effect. On the contrary, to prevent the harmony effect coming into play, we enlarge the single output to the aggregated outputs $\sum_{j \in J} Y_{j}$ for each $\mathrm{DMU}_{j}$ in $\mathrm{J}$ but without changing its input profile, after which we provide a common platform where each mergering DMU has an identical production size to estimate the harmony effect measure. Therefore, to some extent the input mix is avoided when we calculate the size effect measure, and also the size effect does not come into play when we calculate the harmony effect measure. From this perspective, the proposed approach extends the BW framework to wider applications, where DMUs may have different production sizes.

\section{Application to City Commercial Banks in China}

A comprehensive numerical application is provided here to illustrate our proposed models. We consider the mergers of Commercial City Banks (CCBs) in China. Due to the regulation proposed by China Banking Regulatory Commission (CBRC), only 
CCBs with good operating status are allowed to merger. We selected the top 20 competitive CCBs in 2012 as the candidate DMUs. We also used the same inputs and outputs measures proposed in Wang et al. (2014) and Shi et al. (2017) without considering the network structure and the undesirable output (i.e., bad loan). As a result, here are two inputs and two outputs: inputs includes fixed assets $\left(x_{1}\right)$, which refers to the assets value of physical capital, and employee expenses and other operating expenses $\left(x_{2}\right)$, which refers to the payment to full-time employees and the expenses generated during the operating process of CCBs. Outputs includes non-interest incomes $\left(y_{1}\right)$, which includes fees, commissions, investment and other business incomes, and interest incomes $\left(y_{2}\right)$, which refers to incomes that are primarily derived from loans. The overall dataset is given in Table 2 as below, which is derived from the Bank-scope resource package produced by Bureau Van Dijk (BVD).

Table 2 Input and output data of 20 CCBs in China.

\begin{tabular}{cccccc}
\hline DMUs & Name of CCBs & Fixed assets & Expenses & Non-interest incomes & Interest incomes \\
\hline $\mathbf{1}$ & GUANGZH & 977.2 & 351.5 & 3725.1 & 237.7 \\
$\mathbf{2}$ & JINGCCC & 5472.5 & 2119.3 & 18773.1 & 2015.7 \\
$\mathbf{3}$ & SHENGJG & 1443.0 & 2261.5 & 6759.1 & 93.0 \\
$\mathbf{4}$ & JIUJIANG & 652.3 & 775.3 & 3526.3 & 209.7 \\
$\mathbf{5}$ & NANCHNGC & 619.0 & 166.7 & 2224.7 & 456.8 \\
$\mathbf{6}$ & CHENGDU & 1669.5 & 1152.9 & 5387.3 & 222.0 \\
$\mathbf{7}$ & BAOSHANGC & 2870.5 & 5300.1 & 6176.9 & 586.8 \\
$\mathbf{8}$ & HANGZHOUC & 3187.2 & 1181.2 & 8841.8 & 850.2 \\
$\mathbf{9}$ & HUISHNG & 2456.2 & 1404.4 & 6343.8 & 2853.2 \\
$\mathbf{1 0}$ & HARBINC & 2650.6 & 5918.9 & 6658.9 & 970.1 \\
$\mathbf{1 1}$ & JIANGSU & 6627.7 & 650237.9 & 17602.7 & 2196.7 \\
$\mathbf{1 2}$ & ZHUHCIT & 878.0 & 188.4 & 1616.0 & 173.0 \\
$\mathbf{1 3}$ & TIANJINC & 1822.0 & 1324.1 & 6074.8 & 422.1 \\
$\mathbf{1 4}$ & CHONGS & 1557.0 & 1470.5 & 4144.7 & 490.6 \\
$\mathbf{1 5}$ & DALICITY & 2334.1 & 256800.4 & 5559.2 & 626.6 \\
$\mathbf{1 6}$ & LUOYANGC & 411.8 & 313.3 & 1900.2 & 137.4 \\
$\mathbf{1 7}$ & CHANSHAG & 1095.9 & 329.9 & 2948.1 & 329.3 \\
$\mathbf{1 8}$ & WUHAN & 1219.4 & 1255.1 & 3376 & 966.5 \\
$\mathbf{1 9}$ & NANCY & 952.3 & 927.2 & 2389.5 & 900.1 \\
$\mathbf{2 0}$ & NANJINGC & 2296.9 & 1901.6 & 6511.8 & 707.6 \\
\hline
\end{tabular}

For the illustration purpose, we consider the variable returns to scale (VRS) 
assumption in this application section, and as results $\lambda_{j} \in \Omega$ would be formulated as $\sum_{j=1}^{n} \lambda_{j}=1$ (Banker et al., 1984). Other returns to scale properties can also be investigated in a similar way. Further, for convenience of analysis and without loss of generality, we consider only mergers composed of two city commercial banks (i.e., DMUs). As a result, a total of $190\left(C_{20}^{2}=190\right)$ hypothetical mergers are presented here. However, only 136 hypothetical mergers are found feasible in terms of the merger efficiency score $\left(E^{J}\right)$ calculated through model (2). This could be due to the fact that the outputs of these infeasible mergers with two CCBs are too large to be produced under variable returns to scale (VRS) assumption. In addition, 19 hypothetical mergers would be costly with a merger efficiency score $\left(\mathrm{E}^{\mathrm{J}}\right)$ more than one. Based on the current production possible set constructed by these top $20 \mathrm{CCBs}$, these statistics confirms that there exist considerable potential gains from mergers for these CCBs.

Table 3 Distribution of merger efficiency gains.

\begin{tabular}{cccccc}
\hline Efficiency Interval & $\mathbf{E}^{\mathbf{J}}$ & $\mathbf{T}^{\mathbf{J}}$ & $\mathbf{E}^{* \mathbf{J}}$ & $\mathbf{S}^{\mathbf{J}}$ & $\mathbf{H}^{\mathbf{J}}$ \\
\hline $\mathbf{0 . 5 0 0 0 - 0 . 5 9 9 9}$ & 3 & 7 & & & \\
$\mathbf{0 . 6 0 0 0 - 0 . 6 9 9 9}$ & 20 & 29 & 1 & 1 & \\
$\mathbf{0 . 7 0 0 0 - 0 . 7 9 9 9}$ & 39 & 38 & 1 & & 2 \\
$\mathbf{0 . 8 0 0 0 - 0 . 8 9 9 9}$ & 37 & 30 & 6 & 3 & 6 \\
$\mathbf{0 . 9 0 0 0 - 0 . 9 9 9 9}$ & 18 & 17 & 18 & 15 & 86 \\
$\mathbf{1 . 0 0 0 0 - 1 . 0 9 9 9}$ & 12 & 15 & 62 & 54 & 42 \\
$\mathbf{1 . 1 0 0 0 - 1 . 1 9 9 9}$ & 6 & & 46 & 56 & \\
$\mathbf{1 . 2 0 0 0 -}$ & 1 & & 2 & 7 & \\
\hline Average & 0.8320 & 0.7912 & 1.0558 & 1.0834 & 0.9748 \\
\hline
\end{tabular}

Table 3 shows the distribution of merge efficiency gains for all 136 feasible mergers. It should be noted that the majority of these feasible hypothetical mergers are assigned a merger efficiency score $\left(\mathrm{E}^{\mathrm{J}}\right)$ less than one, so considerable potential gains from mergers are expected for each hypothetical merger. On the contrary, only 19 hypothetical mergers' efficiency score $\left(\mathrm{E}^{\mathrm{J}}\right)$ are more than one, indicating that these hypothetical merger would be costly. An average radial measure of the potential overall gains is 0.8320 , which means that if two of the top 20 competitive CCBs are merged to be a new bank, input savings, an average 0.1680 of the pre-merger aggregated inputs, will be achieved in the production of the pre-merger aggregated 
outputs. This result implies that there are significant merger efficiency gains for all top $20 \mathrm{CCBs}$. Meanwhile, the maximal merger efficiency score appears when SHENGJG is merged with JIUJIANG, where $125.31 \%$ of the aggregated inputs are needed to keep the production of the pre-merger aggregated outputs, whereas $46.80 \%$ of the aggregated inputs are saved when BAOSHANGC is merged with ZHUHCIT, implying that it is the most promising hypothetical merger.

On the contrary, after eliminating the technical inefficiency by learning from best practice individually, only 26 hypothetical mergers obtain a pure merger efficiency score $\left(E^{* J}\right)$ less than one. This finding is consistent with Bogetoft and Wang (2005) that the pure potential gains from mergers are considerable less. This also demonstrates that three fourths of these promising mergers will be better off due to the elimination of technical inefficiency instead of real merger and acquisition activities. Table 3 also shows that there are considerable merger gains in terms of the technical effect. Assuming that all banks can be efficient by learning from best practice individually, the estimated average input saving is $20.88 \%$, which implies that $79.12 \%$ of the aggregated inputs are enough to produce the aggregated outputs. Meanwhile, 15 hypothetical mergers get a technical effect score equaling to one, since at least one merging bank is identified as strongly efficient in the sense of Koopmans efficiency.

We decompose the size effect before the harmony effect, which implies that we will not change the input profile arbitrarily. Table 3 shows a moderate potential gains derived from the harmony effect, whereas input costs in terms of the size effect. We get an efficiency score of 0.9748 for the harmony effect and 1.0834 for the size effect, which correspond with a radial measure 1.0558 for the potential overall gains. All the negative pure potential gains from mergers are derived from the size effect, although some potential gains are available here in terms of the harmony effect. In the full sense, the size effect works against mergers in most cases while the harmony effect does favor mergers, which is consistent with Ray (2004), Bogetoft and Wang (2005), and $\mathrm{Wu}$ et al. (2014). In many applications of mergers, the merging DMUs are so large to lose the favor of returns to scale properties. Further, note that the output 
production sizes of these CCBs vary a lot, ranging from 1616 to 18773.1 and from 93.0 to 2853.2 for non-interest income and interest income, respectively, thus our proposed approach may play a significant role to address the decomposition. More importantly, in this paper we give radial measures based on pre-merger aggregated inputs, which are the same as the potential overall gains.

Table 4 Merger efficiencies of the top 10 most promising mergers

\begin{tabular}{cccccc}
\hline Mergers & $\boldsymbol{E}^{J}$ & $\boldsymbol{T}^{\boldsymbol{J}}$ & $\boldsymbol{E}^{* J}$ & $\boldsymbol{S}^{\boldsymbol{J}}$ & $\boldsymbol{H}^{\boldsymbol{J}}$ \\
\hline $7+12$ & 0.5320 & 0.5633 & 0.9444 & 1.0055 & 0.9393 \\
$12+15$ & 0.5739 & 0.6126 & 0.9368 & 0.9947 & 0.9418 \\
$12+14$ & 0.5921 & 0.6359 & 0.9311 & 0.9948 & 0.9360 \\
$7+17$ & 0.6096 & 0.5859 & 1.0405 & 1.0888 & 0.9556 \\
$7+15$ & 0.6234 & 0.5459 & 1.1420 & 1.1419 & 1.0000 \\
$7+16$ & 0.6265 & 0.5802 & 1.0798 & 1.0836 & 0.9965 \\
$7+14$ & 0.6339 & 0.5471 & 1.1587 & 1.1587 & 1.0000 \\
$10+12$ & 0.6463 & 0.6780 & 0.9532 & 0.9845 & 0.9683 \\
$5+7$ & 0.6518 & 0.6051 & 1.0772 & 1.1145 & 0.9665 \\
$7+19$ & 0.6539 & 0.6396 & 1.0224 & 1.0223 & 1.0000 \\
\hline
\end{tabular}

To further explore the most promising and costly mergers, Table 4 lists 10 hypothetical mergers that obtain the lowest $\mathrm{E}^{\mathrm{J}}$ scores and Table 5 lists another 10 hypothetical mergers with the highest $\mathrm{E}^{\mathrm{J}}$ scores. These efficiency scores, $\mathrm{E}^{\mathrm{J}}$ in Table 4, imply considerable improvement potentials from these hypothetical mergers. The merger of $\mathrm{DMU}_{12}\left(\right.$ ZHUHCIT) and $\mathrm{DMU}_{14}($ CHONGS), for example, brings an input saving of $40.79 \%$, and even if the two banks are adjusted to be strongly efficient, an input saving of $6.89 \%$ is still possible. Among these most promising mergers, the technical effect does make a significant contribution, accounting for almost all of the merger efficiency gains. Although these mergers are the top 10 most promising mergers, the size effect works against mergers in most cases (6 out of 10), and even obtains a very high size effect measure, since these merging DMUs are too large to lose the favor of returns to scale properties. Furthermore, we estimate a low harmony effect measure, which implies that the input reallocation takes us into a more productive direction and that production direction brings considerable improvement potentials.

Table 5 Merger efficiencies of the top 10 most costly mergers

\begin{tabular}{llllll}
\hline Mergers & $\boldsymbol{E}^{J}$ & $\boldsymbol{T}^{J}$ & $\boldsymbol{E}^{* J}$ & $S^{J}$ & $\boldsymbol{H}^{J}$ \\
\hline
\end{tabular}




\begin{tabular}{cccccc}
\hline $3+4$ & 1.2531 & 1.0000 & 1.2531 & 1.2531 & 1.0000 \\
$1+5$ & 1.1699 & 1.0000 & 1.1699 & 1.1718 & 0.9984 \\
$4+16$ & 1.1373 & 1.0000 & 1.1373 & 1.1542 & 0.9854 \\
$4+5$ & 1.1253 & 1.0000 & 1.1253 & 1.2066 & 0.9326 \\
$3+16$ & 1.1216 & 1.0000 & 1.1216 & 1.1513 & 0.9742 \\
$1+3$ & 1.1125 & 1.0000 & 1.1125 & 1.1846 & 0.9391 \\
$1+4$ & 1.1062 & 1.0000 & 1.1062 & 1.2463 & 0.8876 \\
$3+5$ & 1.0816 & 1.0000 & 1.0816 & 1.1716 & 0.9232 \\
$5+17$ & 1.0750 & 0.8456 & 1.2713 & 1.2713 & 1.0000 \\
$5+16$ & 1.0750 & 1.0000 & 1.0750 & 1.2071 & 0.8906 \\
\hline
\end{tabular}

In the most costly mergers of Table 5, many additional inputs are needed to keep the production of pre-merger aggregated outputs. Gains derived from the elimination of technical inefficiency are very few, since most merging DMUs are strongly efficient. Assuming that technical inefficiencies have been dealt with, many additional inputs are still needed, and even more than that of $\mathrm{E}^{\mathrm{J}}$. There exist considerable potential gains in terms of the harmony effect, as shown in the last column in Table 5; however, the negative size effect is so heavy that it will prevail the harmony effect and the potential overall gains will only reveal diseconomies of mergers. Furthermore, the size effect works heavily against all the top 10 most costly mergers, with the largest score for $S^{\mathrm{J}}$ being 1.2713 and the least being 1.1513. The loss from the size effect is so critical that it is disadvantageous to merge for these hypothetical mergers.

\section{Conclusions and direction for future research}

In this paper, we develop an alternative approach to decompose the potential gains from mergers. It suggests using strongly efficient projections to measure the efficiency gains. To decompose the technical effect and pure potential gains, we calculate the technical effect measure directly, and all the remaining gains are attributed to the pure potential gains. It finds that if the aggregated projected inputs are proportional to the pre-merger aggregated inputs the proposed approach gives results the same as that of the $\mathrm{BW}$ approach, and if not the proposed approach is till reasonable. Further, this paper provides a practical approach to avoid the mix of size effect and harmony effect when the output production sizes are very different for the original DMUs. We calculate measures of these three effects based on the pre-merger 
aggregated inputs, and it is consistent with the potential overall gains that are decomposed. In addition, we compare the proposed approach with BW approach to highlight some properties, and use a real application to commercial city banks (CCBs) in China to illustrate the usefulness of the proposed approach. By this paper we extend the decomposition framework proposed by BW to wider applications.

Through the real application to City Commercial Banks (CCBs) in China, we could conclude that, (1) there exist considerable potential gains from these hypothetical mergers consisting of the top $20 \mathrm{CCBs}$. However, after the elimination of technical inefficiencies, the majority of these merger efficiency gains disappeared. Therefore, (2) the main effect on potential gains from mergers is supposed to derive from the technical effect. Also, (3) the harmony effect regarding the resource integration will have a significant positive influence on the merger efficiency gains. Lastly, (4) the size effect works against most hypothetical mergers, indicating that it is worthless to participate in full-size mergers.

A drawback of this paper is that the proposed approach calculates the technical effect measure before size effect and harmony effect, and the harmony effect measure is calculated at the full size where the size effect measure has already been calculated, thus the estimated potential gains associated with these three effects depend heavily on the decomposing sequence. Hence, how to develop a non-sequential approach to separate these three effects is a topic for future research. Other future research may apply the proposed approach to practical questions of real mergers and acquisitions. This would be of vital importance for practitioners and managerial applications.

\section{Acknowledgements}

The authors would like to thank two anonymous reviewers and the Editor of Journal of the Operational Research Society for their helpful comments and suggestions. This research was financially supported by the Youth Innovation Promotion Association of Chinese Academy of Sciences, and the National Natural Science Foundation of China (No. 71271196 and 71671172).

\section{Appendix A}




\section{The BW decomposition}

Bogetoft and Wang (2005) suggested to decompose the potential overall gains $\left(E^{J}\right)$ into technical effect $\left(T^{J}\right)$, harmony effect $\left(H^{J}\right)$ and size effect $\left(S^{J}\right)$, namely

$$
E^{J}=T^{J} \cdot H^{J} \cdot S^{J}
$$

In addition, the product of size effect and harmony effect is defined as the pure potential gains from mergers.

$$
E^{* J}=H^{J} \cdot S^{J}
$$

To do the decomposition, BW used model (A3) to estimate the pure potential gains

$$
\begin{array}{ll}
E^{* J}= & \operatorname{Min} E \\
\text { s.t. } & \sum_{j=1}^{n} \lambda_{j} X_{j} \leq E \cdot \sum_{j \in J} \theta_{j}^{*} X_{j} \\
& \sum_{j=1}^{n} \lambda_{j} Y_{j} \geq \sum_{j \in J} Y_{j} \\
& \lambda_{j} \geq 0, \lambda_{j} \in \Omega, j=1, \ldots, n .
\end{array}
$$

In model (A3), $\theta_{j}^{*}$ is the efficiency score of $D M U_{j}$ calculated by model (1), and $\left(\theta_{j}^{*} X_{j}, Y_{j}\right)$ is the weakly projection of $D M U_{j}$. In this way, the aggregated projections can be expressed as $\left(\sum_{j \in J} \theta_{j}^{*} X_{j}, \sum_{j \in J} Y_{j}\right)$. Accordingly, $E^{* J}$ indicates the further optimal proportional reduction on $\sum_{j \in J} \theta_{j}^{*} X_{j}$. As the pure potential gains are those that associate with the residuals of the potential overall gains when the individual technical inefficiency is eliminated, in model (A3) BW captured the pure potential gains by asking the maximal proportional reduction on the aggregated projected inputs $\sum_{j \in J} \theta_{j}^{*} X_{j}$.

According to Formula (A1) and (A2), the technical effect $\left(\mathrm{T}^{\mathrm{J}}\right)$ is calculated by

$$
T^{J}=E^{J} / E^{* J}
$$

Afterwards, BW used a following model to calculate the harmony effect $\left(\mathrm{H}^{\mathrm{J}}\right)$

$$
\begin{aligned}
H^{J}= & \operatorname{Min} H \\
\text { s.t. } & \sum_{j=1}^{n} \lambda_{j} X_{j} \leq H \cdot \alpha \sum_{j \in J} \theta_{j}^{*} X_{j} \\
& \sum_{j=1}^{n} \lambda_{j} Y_{j} \geq \alpha \sum_{j \in J} Y_{j} \\
& \lambda_{j} \geq 0, \lambda_{j} \in \Omega, j=1, \ldots, n,
\end{aligned}
$$


where $\alpha=|J|^{-1} \in(0,1]$ is a scalar that defines the activity level at which the harmony effect measure is calculated, and $|\mathbf{J}|$ is the number of DMUs in J. It is clear that the harmony effect is associated with the input reallocation and output mixture among DMUs with similar size, thus in model (A5), the harmony effect is captured by examining how much of the average inputs could be saved in the production of the average outputs.

Finally, the size effect is calculated by model (A6):

$$
\begin{array}{ll}
S^{J}= & \operatorname{Min} S \\
\text { s.t. } & \sum_{j=1}^{n} \lambda_{j} X_{j} \leq S \cdot H^{J} \sum_{j \in J} \theta_{j}^{*} X_{j} \\
& \sum_{j=1}^{n} \lambda_{j} Y_{j} \geq \sum_{j \in J} Y_{j} \\
& \lambda_{j} \geq 0, \lambda_{j} \in \Omega, j=1, \ldots, n .
\end{array}
$$

The size effect is associated with the change of sizes of merging DMUs, thus it can be calculated by asking how much of the aggregated inputs could be saved by operating at the full size rather than the average size.

\section{Appendix B}

Theorem 1. The pure potential gains measure based on weakly efficient projections is no more than that based on strongly efficient projections, that is, $E^{* J} \leq \hat{E}^{* J}$.

Proof: For efficiency scores $\theta_{j}^{*}$ and non-positive slacks $\delta_{j}^{*}=\left(\delta_{1 j}^{*}, \ldots, \delta_{m j}^{*}\right)^{T}(j \in J)$, it is easy to verify that $\theta_{j}^{*} X_{j}-\delta_{j}^{*} \leq \theta_{j}^{*} X_{j}$. And consequently, we have

$$
\sum_{j \in J}\left(\theta_{j}^{*} X_{j}-\delta_{j}^{*}\right) \leq \sum_{j \in J} \theta_{j}^{*} X_{j}
$$

Model (B2) is used to calculate the pure potential gains based on weakly efficient projections $\left(E^{* J}\right)$ :

$$
\begin{array}{ll}
E^{* J}= & \operatorname{Min} E \\
\text { s.t. } & \sum_{j=1}^{n} \lambda_{j} X_{j} \leq E \cdot \sum_{j \in J} \theta_{j}^{*} X_{j} \\
& \sum_{j=1}^{n} \lambda_{j} Y_{j} \geq \sum_{j \in J} Y_{j} \\
& \lambda_{j} \geq 0, \lambda_{j} \in \Omega, j=1, \ldots, n .
\end{array}
$$

Model (B3) is used to calculate the pure potential gains based on weakly efficient projections $\left(\hat{E}^{* J}\right)$ : 


$$
\begin{aligned}
\hat{E}^{* J}= & \operatorname{Min} E \\
\text { s.t. } & \sum_{j=1}^{n} \lambda_{j} X_{j} \leq E \cdot \sum_{j \in J}\left(\theta_{j}^{*} X_{j}-\delta_{j}^{*}\right) \\
& \sum_{j=1}^{n} \lambda_{j} Y_{j} \geq \sum_{j \in J} Y_{j} \\
& \lambda_{j} \geq 0, \lambda_{j} \in \Omega, j=1, \ldots, n .
\end{aligned}
$$

By comparing model (B2) and (B3), we find that any feasible solution to model (B3), denoted as $\left(\hat{\lambda}_{j}, j=1, \ldots, n ; \hat{E}\right)$, is also feasible solution to model (B2), as it satisfies all constraints in model (B2).

Hence, the feasible region of model (B2) is no smaller than that of model (B3). Then, the optimal objective function to model (B2) is no more than that of model (B3), namely, $E^{* J} \leq \hat{E}^{* J}$

\section{Appendix C}

We demonstrate this point by contradiction.

Firstly, we assume that it holds $\left(E^{J} / E^{* J}\right) \sum_{j \in J} X_{j} \geq \sum_{j \in J}\left(\theta_{j}^{*} X_{j}-\delta_{j}^{*}\right)$. Based on $\sum_{j \in J}\left(\theta_{j}^{*} X_{j}-\delta_{j}^{*}\right) \neq k \sum_{j \in J} X_{j}, k \in(0,1]$, it would be

$$
\left(E^{J} / E^{* J}\right) \sum_{j \in J} X_{j}>\sum_{j \in J}\left(\theta_{j}^{*} X_{j}-\delta_{j}^{*}\right) .
$$

Then, we have

$$
E^{J} \sum_{j \in J} X_{j}>E^{* J} \sum_{j \in J}\left(\theta_{j}^{*} X_{j}-\delta_{j}^{*}\right)
$$

The potential overall gains $\left(E^{J}\right)$ can be calculated by model (C3):

$$
\begin{array}{ll}
E^{J}= & \operatorname{Min} E \\
\text { s.t. } & \sum_{j=1}^{n} \lambda_{j} X_{j} \leq E \cdot \sum_{j \in J} X_{j} \\
& \sum_{j=1}^{n} \lambda_{j} Y_{j} \geq \sum_{j \in J} Y_{j} \\
& \lambda_{j} \geq 0, \lambda_{j} \in \Omega, j=1, \ldots, n .
\end{array}
$$

The pure potential gains $\left(E^{* J}\right)$ can be calculated by model (C4):

$$
E^{* J}=\operatorname{Min} E
$$

$$
\begin{array}{ll}
\text { s.t. } & \sum_{j=1}^{n} \lambda_{j} X_{j} \leq E \cdot \sum_{j \in J}\left(\theta_{j}^{*} X_{j}-\delta_{j}^{*}\right) \\
& \sum_{j=1}^{n} \lambda_{j} Y_{j} \geq \sum_{j \in J} Y_{j} \\
& \lambda_{j} \geq 0, \lambda_{j} \in \Omega, j=1, \ldots, n .
\end{array}
$$

Through model (C3) and (C4), we can find that it is false for 
$E^{J} \sum_{j \in J} X_{j}>E^{* J} \sum_{j \in J}\left(\theta_{j}^{*} X_{j}-\delta_{j}^{*}\right)$, otherwise, the $\mathrm{E}^{\mathrm{J}}$ in model (C3) can be further minimized, which is contrary to the fact that $\mathrm{E}^{\mathrm{J}}$ is the optimal objection function.

So, it would be contrary to formula (C1). Then, the assumption is not established.

Therefore, the relation $\left(E^{J} / E^{* J}\right) \sum_{j \in J} X_{j} \geq \sum_{j \in J}\left(\theta_{j}^{*} X_{j}-\delta_{j}^{*}\right)$ is not held.

\section{Appendix D}

Theorem 3. If $\sum_{j \in J}\left(\theta_{j}^{*} X_{j}-\delta_{j}^{*}\right)=k \sum_{j \in J} X_{j}, k \in(0,1]$, then $E^{* J}=\hat{E}^{* J}, T^{J}=\hat{T}^{J}$.

Proof: If $\sum_{j \in J}\left(\theta_{j}^{*} X_{j}-\delta_{j}^{*}\right)=k \sum_{j \in J} X_{j}, k \in(0,1]$, then

$$
\hat{T}^{J}=\operatorname{Min}\left\{T \in R^{+} \mid \sum_{j \in J}\left(\theta_{j}^{*} X_{j}-\delta_{j}^{*}\right) \leq T \cdot \sum_{j \in J} X_{j}\right\}=k \quad \text { and } \quad \hat{E}^{* J}=E^{J} / k .
$$

The potential overall gains $\left(E^{J}\right)$ can be calculated by model (D1):

$$
\begin{aligned}
E^{J}= & \operatorname{Min} E \\
\text { s.t. } & \sum_{j=1}^{n} \lambda_{j} X_{j} \leq E \cdot \sum_{j \in J} X_{j} \\
& \sum_{j=1}^{n} \lambda_{j} Y_{j} \geq \sum_{j \in J} Y_{j} \\
& \lambda_{j} \geq 0, \lambda_{j} \in \Omega, j=1, \ldots, n .
\end{aligned}
$$

BW used model (D2) to calculate the pure potential gains $\left(E^{* J}\right)$ :

$$
\begin{array}{ll}
E^{* J}= & \operatorname{Min} E \\
\text { s.t. } & \sum_{j=1}^{n} \lambda_{j} X_{j} \leq E \cdot k \sum_{j \in J} X_{j} \\
& \sum_{j=1}^{n} \lambda_{j} Y_{j} \geq \sum_{j \in J} Y_{j} \\
& \lambda_{j} \geq 0, \lambda_{j} \in \Omega, j=1, \ldots, n .
\end{array}
$$

Then, we have $E^{J}=E^{* J} \cdot k$ and $T^{J}=E^{J} / E^{* J}=k$.

Therefore, $E^{* J}=\hat{E}^{* J}, T^{J}=\hat{T}^{J}$.

\section{References}

Andersen, P., Petersen, N.C. (1993). A procedure for ranking efficient units in data envelopment analysis. Management Science, 39(10), 1261-1264.

Bagdadioglu, N., Price, C.W., Weyman-Jones, T. (2007). Measuring potential gains from mergers among electricity distribution companies in Turkey using a non-parametric model. The energy journal, 28(2), 83-110.

Banker, R.D., Charnes, A., Cooper, W.W. (1984). Some models for estimating technical and scale inefficiencies in data envelopment analysis. Management Science, 30(9), 1078-1092.

Banker, R.D., Cooper, W.W., Seiford, L.M., Zhu, J. (2011). Returns to scale in DEA. In: Cooper, W.W., Seiford, L.M., Zhu, J. (eds). Handbook on data envelopment 
analysis. Springer, Berlin, 41-70.

Blancard, S., Boussemart, J.P., Chavas, J.P., Leleu, H. (2016). Potential gains from specialization and diversification further to the reorganization of activities. Omega, 63, 60-68.

Blancard, S., Boussemart, J.P., Leleu, H. (2011). Measuring potential gains from specialization under non-convex technologies. Journal of the Operational Research Society, 62(10), 1871-1880.

Bogetoft, P., Thorsen, B.J., Strange, N. (2003). Efficiency and merger gains in the Danish forestry extension service. Forest Science, 49(4), 585-595.

Bogetoft, P., Wang, D. (2005). Estimating the potential gains from mergers. Journal of Productivity Analysis, 23(2), 145-171.

Charnes, A., Cooper, W.W., Rhodes, E. (1978). Measuring the efficiency of decisionmaking units. European Journal of Operational Research, 2(6), 429-444.

Kristensen, T., Bogetoft, P., Pedersen, K.M. (2010). Potential gains from hospital mergers in Denmark. Health Care Management Science, 13(4), 334-345.

Lo, F.Y., Chien, C.F., Lin, J.T. (2001). A DEA study to evaluate the relative efficiency and investigate the district reorganization of the Taiwan power company. IEEE Transactions on Power Systems, 16(1), 170-178.

Peyrache, A. (2013). Industry structural inefficiency and potential gains from mergers and break-ups: A comprehensive approach. European Journal of Operational Research, 230(2), 422-430.

Ray, S.C. (2004). Data envelopment analysis: theory and techniques for economics and operations research. Cambridge: Cambridge University Press. (pp. 189-208).

Shi, X., Li, Y., Emrouznejad, A., Xie, J. Liang, L., 2017. Estimation of potential gains from bank mergers: A novel two-stage cost efficiency DEA model. Journal of the Operational Research Society, 68(9), 1045-1055.

Wang, K., Huang, W., Wu, J., Liu, Y.N. (2014). Efficiency measures of the Chinese commercial banking system using an additive two-stage DEA. Omega, 44, 5-20.

Wu, D.D., Zhou, Z., Birge, J.R. (2011). Estimation of potential gains from mergers in multiple periods: a comparison of stochastic frontier analysis and Data Envelopment Analysis. Annals of Operations Research, 186(1), 357-381.

Wu, D.D., Birge, J.R. (2012). Serial chain merger evaluation model and application to mortgage Banking. Decision Sciences, 43(1), 5-36.

Wu, D.D., Luo, C., Wang, H., Birge, J.R. (2014). Bi-level programming merger evaluation and application to banking operations. Production and Operations Management, 25(3), 498-515. 